\title{
Ein Schaufenster für die Öffentlichkeit
}

\author{
Das neugestaltete Medizinhistorische Museum der Universität Zürich \\ von Christoph Mörgeli
}

\section{ZuSAMMENFASSUNG}

Ende 1990 hat das Medizinhistorische Museum der Universität Zürich nach jahrzehntelangem wenig besucherfreundlichem Dasein im Uni-Turm einen neuen Standort gefunden. Das Museum präsentiert sich nun in völlig neuer Gestalt im Hochparterre des Gebäudes Rämistrasse 69 (alte Physiologie). Der Konservator beschreibt die längere Standortsuche, die über fünfjährige Planungs- und Realisierungszeit, die inhaltiche Museumskonzeption und deren gestalterische Umsetzung.

Am 27. November 1990 übergab der Baudirektor des Kantons Zürich das renovierte Universitätsgebäude Rämistrasse 69 in einem feierlichen Akt an den Erziehungsdirektor. Am 6. Dezember erfolgte im Beisein von über 500 geladenen Gästen die offizielle Eröffnung des Medizinhistorischen Museums, das im Hochparterre des Gebäudes in vollkommen neuer Gestaltung einen dauernden Platz gefunden hat. Mit diesen Ereignissen war ein Planungsund Realisierungsprozess abgeschlossen, der insgesamt über fünf Jahre beansprucht hatte. Die international bedeutende Zürcher Objektsammlung, die einzige ihrer Art in der Schweiz und eine der wenigen überhaupt, kann damit endlich angemessen präsentiert, erklärt und in den grösseren kulturgeschichtlichen Zusammenhang gestellt werden.

\section{Schwierige Standortsuche}

Der frühere Museumsstandort im vierten Turmgeschoss des UniversitätsKollegiengebäudes an der Rämistrasse 71 konnte kaum jemanden befriedigen. Zu kompliziert gestaltete sich der Zugang zu den Museumsräumen, zu gross war die Hemmschwelle für eine breite Öffentlichkeit, sich durch die Universität bis in die Turmgemächer durchzufragen. Immer wieder haben die Museumsverantwortlichen auf diesen unglücklichen Umstand aufmerk- 
sam gemacht. Schon Bernhard Milt, erster Inhaber des medizinhistorischen Lehrstuhls in Zürich, unternahm zwei vergebliche Versuche, die Objektsammlung zu verlegen. Das ehemalige Bibliotheksgebäude Martin Bodmers an der Bederstrasse 28 - als Standort des Museums für geeignet befunden wurde 1950 anderweitig vermietet. 1953 verwarf der Zürcher Kantonsrat einen Kredit zur Unterbringung des Medizinhistorischen Museums im Haus Belmont an der Rämistrasse 67 und befürwortete lediglich einen bescheidenen Ausbau der Turmgeschosse. Während die bürgerlichen Fraktionen die Vorlage unter Hinweis auf die Bedürfnisse von Wissenschaft und Publikum unterstützten, hielten die linken Volksvertreter eine verbesserte Ausstellung des Sammlungsgutes für überflüssig. Anfang 1981 schien sich mit der Diskussion um die Nutzung des alten Physiologiegebäudes an der Rämistrasse 69 eine neue Möglichkeit zu eröffnen. Der damalige Institutsdirektor Huldrych M.Koelbing empfahl bei den zuständigen Stellen mit Nachdruck die Berücksichtigung der Medizingeschichte, falls das Gebäude erhalten werden könne. Obwohl das Haus dank entschiedener Opposition von Stadtrat und Heimatschutzkreisen dem vorgesehenen Abbruch knapp entging, stand das Museum in der Planung vorerst nicht im Vordergrund. 1982 führte Konservator Urs Boschung intensive Gespräche mit der Stadt Zürich; die Übersiedlung des Medizinhistorischen Museums in die Liegenschaft Stüssihofstatt 9, mitten in der Altstadt, lag in greifbarer Nähe. Dem Nachteil der recht engen Räumlichkeiten standen die Vorteile einer zentralen Lage und der Geschichtsträchtigkeit des Hauses gegenüber : Seit dem 16. Jahrhundert diente der «Schwarze Garten» den Scherern und Badern der Schmiedenzunft, 1782 bis 1833 dem Medizinisch-chirurgischen Institut als Unterkunft. Die Stadt Zürich lehnte aber eine Vermietung für museale Zwecke ab, da die Liegenschaftenverwaltung dem Erhalten von Wohnraum Priorität einräumte.

\section{Planung im Gebäude Rämistrasse 69}

1983 zeichnete sich die universitätsinterne Unterbringung des Medizinhistorischen Museums im Hochparterre des zu renovierenden Gebäudes Rämistrasse $69 \mathrm{ab}$. Im weiteren waren auch die Raumansprüche von technischen Diensten, PC-Arbeitsräumen für Studenten, Soziologischem Institut und gemeinsamem Lehrbereich zu befriedigen. Mit dem Sanierungsauftrag und der Museumsgestaltung wurde der Zürcher Architekt Hans Howald betraut, der an der EXPO 1964 beträchtliche Ausstellungserfahrung gesammelt 
hatte. Urs Boschung erarbeitete für die zur Verfügung stehenden Räume ein Grobkonzept. Eine Verzögerung in den Planungsarbeiten trat anlässlich des Konservatorenwechsels vom Früjahr 1985 ein; Boschungs Nachfolger Christoph Mörgeli musste sich erst in das Projekt und überhaupt in die Medizingeschichte einarbeiten. 1987 verstarb Architekt Hans Howald - dem wir entgegen der Vorstellung der universitären Raumkoordination die Zuteilung eines sechsten Raumes verdanken -, worauf sein Mitarbeiter Rolf Wolfensberger die architektonische Leitung der Gebäudesanierung übernahm. In langwierigen, zuweilen hart geführten Diskussionen mussten der Konservator mit seinem inhaltlich-wissenschaftlichen Konzept und der Architekt mit seinen gestalterischen Vorstellungen eine gemeinsame Basis finden. Es war nicht immer leicht, Architekt und Vertreter der kantonalen Abteilung für Universitätsbauten und der universitären Raumkoordination zu überzeugen, dass sich bei einer Ausstellungsgestaltung die Form nach dem zu vermittelnden Inhalt zu richten hat. Dank Mitwirkung des Ausstellungsdienstes der Universität und von Hans Konrad Schmutz als externem musealem Berater konnte das Institut seine Vorstellungen schliesslich durchsetzen oder doch akzeptable Kompromisse erreichen. Dem Konservator ging es darum, die (nicht vorgebildeten) Besucher in einem didaktisch aufgebauten Lehrpfad durch die Medizingeschichte zu führen, einen Lernvorgang zu erzeugen und die gezeigten Objekte mit klaren Aussagen und Grafiken zu erläutern; der Betrachter sollte mit den mitunter erschreckenden Apparaten und Instrumenten nicht allein gelassen werden. Die Knappheit des Raumes (insgesamt $525 \mathrm{~m}^{2}$ ) zwang zur Auswahl und Reduzierung der Zahl der Objekte, was sich für den Konservator zuweilen als schmerzlich, von didaktischen Gesichtspunkten aber meist als nützlich erwies. Architektonisch gelangte man schliesslich zum konsequenten Erhalten der bestehenden Raumstruktur von 1885; einzig für die Schauszenarien und für den Medienraum wurden neue Gipswände eingezogen. Die notwendigen statischen Eingriffe geschahen in einfachster, möglichst diskreter Art. Vorgeschlagene Formelemente, so interessant sie vom architektonischen Gesichtspunkt sein mochten, mussten abgelehnt werden, wenn sie mit der Ausstellungsaussage in keinen Zusammenhang zu bringen waren. Die Vitrinen wurden einem Anforderungskatalog gemäss speziell für das Medizinhistorische Museum angefertigt. Es handelt sich um Prismen von $1 \mathrm{~m}$ Breite, $0,5 \mathrm{~m}$ Tiefe und 2,2 $\mathrm{m}$ Höhe aus schwarz gebeiztem Eichenholz mit verschliessbarer Glastüre und Beleuchtung im Kopfteil. Für die Texte stehen analoge, 0,5 m breite Prismen zur Verfügung. Die belichteten Vitrinen ergänzt eine gezielte Be- 
leuchtung freistehender Objekte durch Spots und ein indirektes, gedämpftes Raumlicht. Aus konservatorischen Gründen kann das Tageslicht nicht direkt in die Räume eingelassen werden. Helle Textilrouleaus schützen die Objekte vor UV-Strahlung, ohne den räumlichen Eindruck der mächtigen Rundbogenfenster zu verdecken. Dank einem Hohlraumboden können bei Veränderung der Ausstellung alle notwendigen elektrischen Installationen (Strom, Audio- und Videoverbindungen) erstellt bzw. ergänzt werden.

\section{Ein kulturgeschichtliches Museum}

Die Universitätsmuseen bilden gewissermassen die Fenster im Gemäuer, hinter dem sich der Lehr- und Forschungsbetrieb für die Augen der Allgemeinheit oftmals verbirgt. Als hauptsächlich anzusprechende Zielgruppe stand von Anfang an eine breite Öffentlichkeit fest, was in der Ausstellungsgestaltung zu einfachen, allgemeinverständlichen Aussagen zwang. Gerade bei einem Medizinhistorischen Museum durfte davon ausgegangen werden, dass Fragen von Gesundheit und Krankheit jeden Menschen ganz unmittelbar betreffen. Es ergab sich die Chance, an einem Ort der entkrampften Begegnung mit der geschichtlichen Entwicklung der Medizin mögliche Ängste, Fehl- oder Vorurteile abzubauen. Weitere Zielgruppen sind erfahrungsgemäss medizinisch speziell interessierte oder betroffene Laien sowie Vertreter und Auszubildende der verschiedenen Medizinalberufe (Ärzte, Zahnärzte, Medizinstudenten, Krankenpflege- und Laborschüler usw.).

Das Medizinhistorische Museum ist trotz etlicher Exponate von höchstem künstlerischem Interesse kein Kunstmuseum und auch kein Ort komplizierter Technik- oder Wissenschaftsgeschichte. Als kulturgeschichtliches Museum zeigt es die Bemühungen um die Heilkunst als Teil des menschlichen Wirkens im Rahmen der Geschichte auf. Dabei sollen Eindrücke eines Gruselkabinetts vermieden werden, zeugen doch selbst die wenig zimperlich anmutenden Operationsmethoden der Handwerkschirurgen vom ernsten, ehrlichen Bemühen früherer Menschen, an deren Denken und Tun nicht heutige Massstäbe angelegt werden dürfen. Wenn die Besucher die Ausstellung mit dem positiven Gefühl einer heutigen Medizin verlassen, der sie sich zuverlässiger als frühere Generationen anvertrauen dürfen, liegt dies durchaus in der Absicht der Museumsgestalter. Das soll indessen nicht heissen, dass problematische Seiten in der neueren Entwicklung des Gesundheitswesens einfach ausgeblendet würden. 


\section{Lehrpfad durch die Medizingeschichte}

Die ständige Ausstellung setzt ein mit der Medizin der «Naturvölker» und dem christlichen Krankheitsverständnis. Im Hauptraum (180 m²) folgt ein geraffter Überblick von der prähistorischen Zeit über die alten und klassischen Hochkulturen bis zum Mittelalter. Die neue Anatomie und Physiologie bilden die Grundlage für die Fortschritte der Chirurgie, die im 19. Jahrhundert eine weitgehende Aufgliederung in Spezialfächer erlebt (Zahn- und Augenheilkunde, Orthopädie, Ohren-Nasen-Hals-Heilkunde, Geburtshilfe und Gynäkologie). Voraussetzungen für die moderne Chirurgie bilden die Verfahren von Anästhesie, Asepsis, Röntgen und Blutersatz. In einem weiteren kleinen Raum $\left(24 \mathrm{~m}^{2}\right)$ wird die Entwicklung der Medikamente von Rohdrogen zu Wirkstoffen erläutert. Auch der Geschichte von Infektionskrankheiten wie Pest, Lepra, Syphilis, Pocken, Tuberkulose, Kinderlähmung und Aids sowie der Labormedizin wird neuerdings breiterer Raum gewährt $\left(72 \mathrm{~m}^{2}\right)$. Schliesslich zeigt der Museumsraum mit den Themen Kranken- und Säuglingspflege, physikalische Therapie und Psychiatrie (34 $\mathbf{m}^{2}$ ) die sich wandelnden Möglichkeiten der Patientenbehandlung im Lauf der Geschichte.

Tritt in der Dauerausstellung die Personen- oder Standesgeschichte bewusst zurück, vergegenwärtigt eine Galerie von Gemälden und Stichen im grosszügig angelegten Entree $\left(90 \mathrm{~m}^{2}\right)$ die bedeutende Rolle zürcherischer Ärzte für die Entwicklung der europäischen Medizin. Periodisch angebotene, zeitlich und thematisch beschränkte Sonderausstellungen in einem entsprechend vielfältig nutzbaren Raum $\left(89 \mathrm{~m}^{2}\right)$ sollen das Museum lebendig erhalten und immer wieder neue Besucherkreise ansprechen. Den Charakter einer Begegnungsstätte unterstreicht der für Klassen, Gruppen und Institutsveranstaltungen freigehaltene Seminarraum $\left(34 \mathrm{~m}^{2}\right)$, wo medizinhistorische Kurse stattfinden und das Gesehene theoretisch aufgearbeitet werden kann.

\section{Finanzierung und Schau-Szenarien}

Die Gesamtaufwendungen der Gebäudesanierung Rämistrasse 69 betrugen rund 12 Millionen Franken; davon entfielen ca. Fr. $750000 .-$ auf die Neugestaltung des Medizinhistorischen Museums. Das Hochbauamt des Kantons Zürich kam für die baulichen Massnahmen sowie für das gesamte Ausstel- 
lungsmobiliar auf. Der Ausstellungsdienst der Universität - bestehend aus dem Leiter, einem Grafiker und einer Fotografin - erbrachte Eigenleistungen im Wert von ca. Fr. 130 000.-. Dem Institut verblieben für Anschaffungen von Objekten und gezielte Restaurierungsarbeiten ca. Fr. 140000.- aus einem Pool der Universitätsmuseen, desgleichen ein Betrag von Fr. 80 000.für gezielt eingesetzte audiovisuelle Mittel: ein kleiner Medienraum erlaubt nun die Vorführung individuell durch die Besucher auszuwählender Videos zu verschiedensten Themen von Medizingeschichte und aktueller Medizin. Nicht durch die öffentliche Hand gedeckt waren der Ausbau von SchauSzenarien, spezielle grafische Arbeiten und der Museumskatalog. Eine vom Rektorat bewilligte Finanzaktion bei Stiftungen, Privaten, Banken, Versicherungen, Krankenkassen sowie Firmen für Pharmazeutika und Medizinalbedarf erbrachte die stattliche Summe von Fr. $272500 .-$. Wir verdanken den grosszügigen Spendern, dass nun mehrere nachgestellte Schau-Szenarien einen wirklichkeitsnahen Einblick in mögliche Schauplätze der Medizingeschichte bieten. Der eindrucksvolle afrikanische Heilkundige präsentiert seine Gerätschaften vor der Inneneinrichtung seines Hauses. Dem verhüllten, schnabelbewehrten Pestarzt des 17. Jahrhunderts nebst Pestsarg steht der nicht minder vorsichtige Pestforscher des beginnenden 20. Jahrhunderts mit seinem mobilen Pestlabor gegenüber. Eine ausgezeichnet erhaltene Apotheke aus dem deutschen Rheinland zeigt den Stand einer geheimnisvoll-exotisch anmutenden Pharmazie im 18. Jahrhundert. Die bäuerliche Appenzeller Kindbettkammer der 1840er Jahre erzählt von den damals üblichen Hausgeburten und der wichtigen Tätigkeit der Hebamme. Ein ärztlicher Praxisraum zeugt von den diagnostischen und therapeutischen Möglichkeiten eines Allgemeinpraktikers vor der letzten Jahrhundertwende. Ein medizinisches Labor zur Untersuchung von Blut, Urin und Stuhl von 1920 ist demjenigen von 1990 gegenübergestellt.

\section{Ausblick und praktische Hinweise}

Einige Monate nach Inbetriebnahme darf die Neukonzeption des Medizinhistorischen Museums als gelungen betrachtet werden. Die Reaktionen des Publikums auf den neuen Standort wie auf die Neugestaltung sind durchwegs positiv. Die wesentlich erweiterten Öffnungszeiten von Dienstag bis Freitag 13-18 Uhr, Samstag und Sonntag 11-17 Uhr (Montag geschlossen) haben zu einer wesentlichen Steigerung der Besucherzahl geführt. An einem 
Wochentag halten sich nach bisherigen Erfahrungen mehrere Dutzend, an den Wochenenden oft über 100 Besucher im Museum auf. Die Nachfrage nach Führungen ist überaus gross; leider lassen sich wegen des knappen Personalbestandes in diesem wichtigen Bereich der Öffentlichkeitsarbeit nicht alle Bedürfnisse befriedigen. Berufsschul- und andere Klassen können leider nicht mehr geführt, die Lehrerinnen und Lehrer aber mit entsprechendem Vorbereitungsmaterial versorgt werden. Bei freiem Eintritt für Einzelpersonen müssen Führungen durch Institutsmitarbeiter verrechnet werden. Bei den erweiterten neuen Öffnungszeiten scheint es zumutbar, Führungen in der Regel nur noch während der offiziellen Zeiten zu gestatten; aus Gründen der Sicherheit sind der individuelle Einlass und die fast «familiäre» Betreuung wie seinerzeit im Turm nicht mehr denkbar. Im Frühjahr 1991 wird der ausführliche, 144 Seiten und 115 Farbabbildungen umfassende Museumskatalog erscheinen. Auch fremdsprachige Begleittexte, Ansichtskarten und Dias für den Verkauf sind in Vorbereitung.

Mit Wechselausstellungen, Dokumentationsangeboten, Führungen im Rahmen der (beschränkten) Möglichkeiten gilt es nun, das Museum nach der Vollendung nicht erstarren zu lassen, sondern lebendig zu erhalten. Eine gute Voraussetzung bietet dazu der neue, geradezu ideale Standort in einem eigentlichen Museenzentrum im Bereich von oberer Rämistrasse/Künstlergasse (Archäologisches, Paläontologisches und Zoologisches Museum). Mit den Möglichkeiten der modernen Ausstellungstechnik kann fortan die medizinhistorische Objektsammlung von Zürich wieder angemessen präsentiert werden - eine Sammlung, der weltweit nur wenige an Vielseitigkeit und Wert gleichkommen. 


\section{Summary}

\section{The Zurich Museum of Medical History in a new setting}

The author describes the long search for a suitable place for the museum as well as the five years of planning, reconstructing and arranging the new display of often unique objects. The idea is to present the history of medicine with a view to lead the visitor to a better understanding of medicine in general and thus to a rather confident attitude towards the medicine of to-day.

\section{Résumé}

\section{Réouverture du Musée d'Histoire de la Médecine de l'Université de Zurich}

Ce fameux musée vient de trouver une nouvelle demeure. Après avoir occupé pendant des décennies des locaux peu accueillants dans la tour du bâtiment principal de l'Université, il se trouve maintenant (depuis décembre 1990) au rez-de-chaussée de l'«ancienne physiologie» (Rämistrasse 69, directement à gauche de l'Université). L'auteur, en sa qualité de conservateur du musée, relate la longue recherche de locaux convenables aussi bien que les efforts de plus de cinq ans qu'il fallait pour établir et réaliser le nouveau projet. Les objets historiques, souvent uniques, sont présentés de sorte que les visiteurs puissent gagner une meilleure compréhension de la médecine en général, ci-inclus la médecine d'aujourd'hui.

Heures d'ouverture : mardi à vendredi $13-18 \mathrm{~h}$, samedi et dimanche $11-17 \mathrm{~h}$, fermé lundi.

Dr. phil. Christoph Mörgeli

Konservator des Medizinhistorischen Museums

Rämistrasse 71

CH-8006 Zürich 